\title{
Topology Density Map for Urban Data Visualization and Analysis
}

(b)

(a)
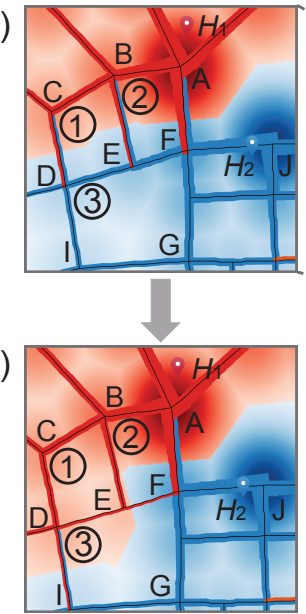

Zezheng Feng, Haotian Li, Wei Zeng, Shuang-Hua Yang, and Huamin Qu

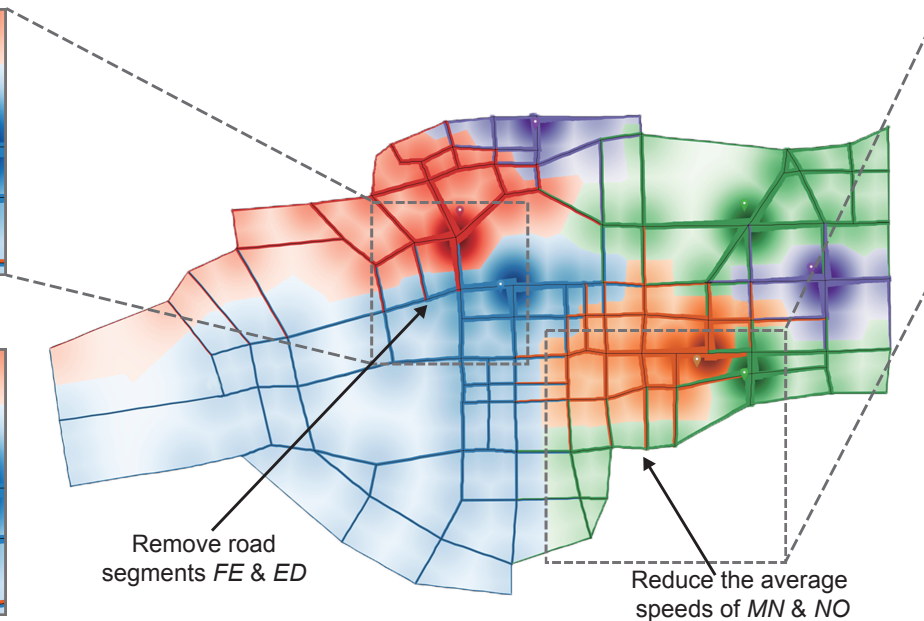

(c)

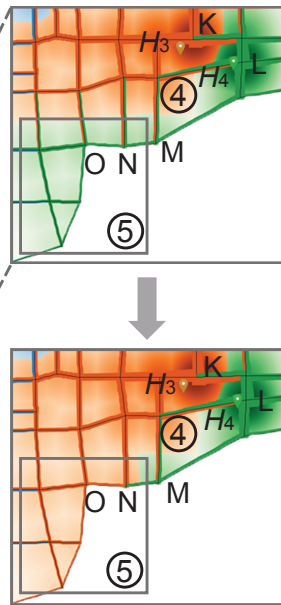

Fig. 1. Analyzing the influence of road topology and traffic conditions on POI accessibility: density distribution before (a) and after (b) removing the selected road segments $F E$ and $E D$; and density distribution before (c) and after (d) slowing down the average speeds of the selected road segments $M N$ and $N O$.

\begin{abstract}
Density map is an effective visualization technique for depicting the scalar field distribution in 2D space. Conventional methods for constructing density maps are mainly based on Euclidean distance, limiting their applicability in urban analysis that shall consider road network and urban traffic. In this work, we propose a new method named Topology Density Map, targeting for accurate and intuitive density maps in the context of urban environment. Based on the various constraints of road connections and traffic conditions, the method first constructs a directed acyclic graph (DAG) that propagates nonlinear scalar fields along 1D road networks. Next, the method extends the scalar fields to a 2D space by identifying key intersecting points in the DAG and calculating the scalar fields for every point, yielding a weighted Voronoi diagram like effect of space division. Two case studies demonstrate that the Topology Density Map supplies accurate information to users and provides an intuitive visualization for decision making. An interview with domain experts demonstrates the feasibility, usability, and effectiveness of our method.
\end{abstract}

Index Terms-Density map, network topology, urban data

\section{INTRODUCTION}

Road network is an important infrastructure in cities. With expansion of cities and evolution of transportation technology, road networks are becoming increasingly intensive and unobstructed. Consequently, two geographically distant locations may have a short commuting time due to road connectivity [2]. Hence, road network is of importance in many real-world applications, including urban planning [39] and urban scaling analysis [26]. For example, traffic management can benefit from forecasting and visualizing the congestion conditions of a city based on vehicle detector data on roads [25]. Moreover, road network also shields an effect on urban data visualization. For an example, a recent work of [51] presented a more accurate edge bundling method

- Z. Feng H. Li and H. Qu are with the Hong Kong University of Science and Technology.E-mail: \{zfengak, haotian.li\}@connect.ust.hk and huamin@cse.ust.hk

- W. Zeng is with Shenzhen Institutes of Advanced Technology, Chinese Academy of Sciences. Wei Zeng is the corresponding author. E-mail: wei.zeng@siat.ac.cn.

- SH. Yang is with Southern University of Science and Technology. E-mail: yangsh@sustech.edu.cn.

Manuscript received xx xxx. 201x; accepted xx xxx. 201x. Date of Publication $x x$ xxx. 201x; date of current version xx xxx. 201x. For information on obtaining reprints of this article, please send e-mail to: reprints@ieee.org. Digital Object Identifier: $x x . x x x x / T V C G .201 x . x x x x x x x$ for urban traffic data, by carefully considering the constraints implied by underlying road networks.

Density map, as an effective visualization technique [14], is widely used in urban analysis, which involves spatial distribution patterns [50], such as anomaly detection [41], risk analysis [33] and air pollution propagation analysis [9]. A density map depicts the continuous distribution of scalar field in a 2D planar space by assigning a unique color to each individual scalar value [16 37]. The scalar field is computed from the premise of the finite observation of the data [37]. The process is referred as density estimation, which can be parametric or nonparametric. Kernel density estimation (KDE) is a common nonparametric model, which typically applies a kernel (e.g., parabolic, Gaussian, and Sigmoid) to the proximity between two locations. The proximity is typically computed as Euclidean distance in a Cartesian coordinate system. Due to its simplicity, KDE has been widely adopted in movement visualization, such as vessel movements [33 46] and flight trails [18, 19].

Nevertheless, the KDE based on Euclidean distance is inappropriate for many urban analyses that should take road networks into consideration, simply because most movements in cities are constrained by road networks [5]. Fig. 1 illustrates a real-world scenario, where domain experts would like to analyze how easy to access points-of-interest (POIs), i.e., the POI accessibility. For accurate accessibility measurement, we shall consider the following properties of a road network when measuring the proximity between two locations: 
- Topological: Locations are connected through roads and intersections, which can be obstructed by obstacles, such as accidents or road repairs. For instance, if the path $(F-E-D)$ in Fig. 11 a $)$ is under repair, the shortest paths from $H_{2}$ to locations in region 1 will be altered, affecting POI accessibility in the region.

- Directional: Unlike the Euclidean distance, the proximity of A $\rightarrow \mathrm{B}$ could be very different from that of $\mathrm{B} \rightarrow \mathrm{A}$. In most cities, a road has two different directions, which can be modeled as two directional edges. For example, when the aforementioned case in Fig. 11a) occurs, the shortest path $(E \rightarrow D)$ changes but that in the other direction $(D \rightarrow E)$ remains the same.

- Temporal variant: The proximity here is typically measured by the access time rather than the physical distance, which depends much on traffic conditions that vary over time. For instance, the POI accessibility in Region 5 is greatly affected by traffic conditions, e.g., the non-peak hours in Fig. 1(c) and the peak hours in Fig. 1. d).

The transportation and geography communities have strived to include road networks in KDE computation, e.g., [7 8]. However, proximity measurement only applies to one-dimensional road networks [40], whilst density map requires scalar field distribution in a 2D planar surface. This limitation yields unintuitive visualizations compared with density map. A new method is required to derive scalar fields outside the road network. We consider the following requirements for such a method: i) correctness, which should accurately coordinate with the complex road topology and the dynamic traffic conditions; and ii) intuitiveness, which should intuitively depict density fields on a 2D planar surface instead of a 1D road network.

In this study, we propose Topology Density Map to fulfill the requirements. The method works as follows: First, we construct a directed acyclic graph (DAG) from each POI to all road intersections, taking road topology and traffic conditions into consideration. Next, we find shortest paths to intersections with minimum access times, and measure the density propagation along the shortest paths. Lastly, we extend density estimation from each intersection to the neighboring regions, and then generate a density map based on the estimated 2D scalar fields. The main contributions of this work are listed as follows:

- The topology density map extends the existing networkconstrained density estimation method from a 1D road network to a 2D planar surface, providing more correct and intuitive density field estimation in an urban area.

- The topology density map forms a weighted Voronoi diagram like effect that reflects the space partition by the POI accessibility. This kind of visualization is especially useful when multiple POIs affect the same region.

- The feasibility and usability of the topology density map are demonstrated by two real-world case studies, and the effectiveness of the method is confirmed by expert feedbacks.

The remaining of this paper is structured as follows: Sec. 2 discuses related works in visual analytics and visualizations for urban data analysis. Sec. 3 summarizes the limitations of existing density estimation methods and provides an overview of our approach. Sec. 4 describes the domain problems and details of preparing input data. Sec.5 5 presents the details of the topology density map construction, and the comparison of the visual effect with network KDE. Sec. 6 demonstrates two case studies conducted on a real-world dataset and the feedbacks from three independent domain experts. Sec.7 7 describes the various considerations of our approach, including the parameter selection, the computing efficiency, and alternative designs, and discusses the limitations and future works. Finally, Sec. 8 concludes this paper.

\section{Related Work}

\subsection{Visual Analytics for Urban Traffic}

Benefiting from the advancement of location sensing technologies, movement data, which characterize moving objects in the location space (where), time space (when), and attribute space (what) [22], become

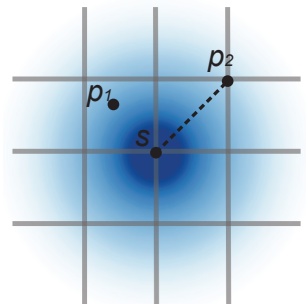

(a) Planar KDE

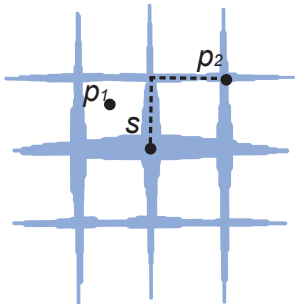

(b) NKDE
Fig. 2. Illustration of density estimation using (a) planar KDE (Euclidean distance), and (b) NKDE (network distance). Planar KDE encodes density fields using colors, while NKDE uses line width.

ubiquitous and abundant [38]. Urban traffic is a specific movement data that record commuter/goods/vehicle movements on road networks in a city. Urban traffic data provide unprecedented information on human activities [1], and visual analytics is an effective tool for analyzing urban traffic [5]. Many visual analytics have been developed for urban traffic in different cities for different purposes, such as to study hidden themes of taxi movements in Shenzhen [6], to make an improvement in the performance for bus route planning in Beijing [44], to stack and visualize trajectory attributes for movements in San Francisco [43], and to explore commuting movements in New York City [11]. Interested readers may refer to the surveys [1 5].

Visual clutter is a main issue in visual analytics for urban traffic, and it is mainly caused by large amounts of traffic data. Many solutions have been proposed to mitigate this problem, such as designing effective filter interactions [23], building efficient query database [11], and developing realistic edge bundling [51]. Among the solutions, density map is an effective visualization technique for depicting scalar fields on a 2D planar surface [33,46]. Many visual analytics adopt a density map to present information in an urban environment. For example, Shen et al. [35] identified hotspots of taxi movements from taxi GPS traces, which can help drivers find optimal paths between a pair of start-end locations. Liu et al. [27] revealed the space visibility from taxi GPS trajectory data, which can assist planners in identifying optimal locations for placing advertisement boards.

Both works adopted density-based epsilon distance algorithms to generate density maps. However, the algorithms can only model the scalar field propagation at local scales, whilst many city-scale urban analyses require considerations of road network and traffic conditions, such as mobility [49] and transportation efficiency analysis [10]. This work aims to fill the gap with a feasible solution for city-scale urban analysis. We develop a new approach to density map construction, namely, Topology Density Map. We show the efficiency of the topology density map in city-scale accessibility analysis.

\subsection{Density Estimation in Urban Analysis}

Scalar fields distributed over space can be visualized as a field schematization that aggregates representatives for local regions based on field data, i.e., density maps [16]. As an effective visualization technique, density maps have been widely adopted in urban analysis [52]. A key step in density map construction is measuring the scalar field distribution in the study area. Many density estimation methods have been proposed. Among them, kernel density estimation (KDE) has been proven efficient and effective [4]. On the basis of proximity measurement, the KDE for urban analysis can be divided in two parts: the density estimation based on the Euclidean distance - planar KDE (Fig.2(a)), and the density estimation based on network proximity network KDE (NKDE) (Fig. 2.b)).

Planar KDE obeys the rule that in a Cartesion coordinate system, straight line is the shortest between two points, i.e., Euclidean distance. Planar KDE is frequently employed when the trajectory position is unconstrained, such as vessel movements [33 46] and flight trails [18. 19]. In cities, however, the method is only suitable for local-scale urban analyses. For example, Grubesic [12] adopted planar KDE in detecting hotspots for crime in a city, and Kloog et al. [21] applied planar KDE 
in studying the relationship between breast cancer cases and artificial lighting at night. For city-scale analyses that involve urban traffic along road networks, planar KDE is no longer suitable. For an example, Zeng et al. [49] studied passenger mobility using the public transportation system in a city, which cannot be depicted using planar KDE.

The NKDE that estimates density based on the proximity along a road network is appropriate for city-scale urban analyses. However, NKDE construction is rather challenging. First, we need to find a suitable proximity measurement on the road network, which could be affected by many factors, such as traffic conditions, street crossings, and obstacles, etc. For instance, Delso et al. [7] considered the impact of street crossings when measuring travel times between positions. Second, we need to develop an efficient algorithm for computing pairwise proximities. For example, Deng et al. [8] constructed a networkconstrained Delaunay triangulation to facilitate the measurement of network proximities between locations. Nevertheless, even when these two challenges are well resolved, density fields are computed as propagation along 1D road networks (Fig. 2 b)), which is less intuitive in comparison with density fields on a 2D planar space (Fig.2 a)).

We develop topology density map that combines the intuitiveness of planar KDE in presenting continuous density fields over space and the correctness of NKDE in considering the road topology and traffic conditions for city-scale urban analysis. Various optimization techniques are designed to facilitate the construction and visual effect of the topology density map.

\section{Requirement Analysis and Method Overview}

Density estimation is the key step in density map generation. This section briefly introduces planar KDE (Sec. 3.1.1) and NKDE (Sec. 3.1.2), followed by a discussion of the requirements when applied to urban data analysis and visualization (Sec. 3.2. An overview of our topology density map is presented at the end (Sec. 3.3.

\subsection{Conventional Density Estimation}

In this work, we regard planar KDE as a density estimation method that adopts kernel functions with Euclidean distance, while NKDE is a special case of planar KDE, with the distance measured on the road network as described in [48]. Both approaches are state-of-the-arts methods for estimating density fields.

\subsubsection{Planar KDE}

Planar KDE is a nonparametric estimation method, i.e., not making use of prior knowledge about the data distribution. Instead, the method studies the data distribution characteristics from the data themselves. Planar KDE simulates a probability distribution curve using a smooth kernel function to fit the observed data points. The method is often used to estimate unknown probabilities, e.g., movement distributions in a 2D space [ $\left.\begin{array}{l|l|l|l}18 & 19 & 33 & 46\end{array}\right]$.

Given a set of observed data points $D:=\left\{x_{i}\right\}$, we can estimate density field $\lambda(s \mid D)$ at location $s$ in a $2 \mathrm{D}$ space as follows:

$$
\lambda(s \mid D)=\sum_{x_{i} \in D} \frac{1}{\pi r^{2}} K\left(\frac{d\left(x_{i}-s\right)}{r}\right)
$$

where $r$ is the kernel radius, which is often called bandwidth, $d(\cdot)$ is the Euclidean distance between point $x_{i}$ and location $s$, and $K$ is a weighting function known as kernel function. Several common kernel functions exist, including parabolic, Gaussian, Sigmoid, and negative exponential functions. The function value typically decreases as the distance increases, coping with the distance-decay effect. Given this property, planar KDE is often employed in spatial analysis, as many geographic phenomenon follow the first law of geography, which states that "everything is related to everything else, but near things are more related than distant things" [42].

\subsubsection{NKDE}

NKDE is usually used to estimate the density fields of road-constrained events on a road network, such as the impacts of traffic accidents. Density fields are measured as linear propagations along a road network.
Given observed data points $D:=\left\{x_{i}\right\}$ and road network $G$, density field $\lambda(s \mid D, G)$ at location $s$ can be estimated as:

$$
\lambda(s \mid D, G)=\sum_{x_{i} \in D} \frac{1}{r} K\left(\frac{d_{G}\left(x_{i}-s\right)}{r}\right)
$$

where parameters $r$ and $K$ represent the same settings with those in planar KDE, while $d_{G}(\cdot)$ denotes the network proximity between $x_{i}$ and $s$. In this sense, the function is only valid for location $s$ on the road network $G$. According to Fig. 2 b), we can only estimate the density field outside the road network at $p_{2}$, but not for $p_{1}$.

\subsection{Requirement Analysis}

Though popular, planar KDE and NKDE fail to fulfill the following requirements when applied to urban data analysis.

R1. Correctness. As discussed above, planar KDE can provide an intuitive representation of the density fields over a 2D space. The method employs Euclidean distance to estimate the density fields within the kernel radius, which however is unsuitable for urban areas. This is because road networks in a city typically exhibit a complex topology and dynamic traffic conditions that should be considered. There may not be a straight connection between two locations, or a straight connection may not be the fastest path due to traffic jams or road maintenance. In such scenarios, people no longer commute via a straight line, but alternatively choose a suitable path according to road connections and current traffic conditions, such as the path between $p_{2}$ and $s$ in Fig. 2 b). Furthermore, in practice, roads in a city are often bidirectional, with median dividers separating roads into opposite directions. On these roads, vehicles cannot cross the road or change directions until they reach turning points. However, planar KDE cannot reflect the bidirectional characteristic when estimating density fields. Therefore, when analyzing urban problems that consider human mobility on road networks, planar KDE will produce an inaccurate density field estimation, generating incorrect visualization that may be detrimental to decision-making.

R2. Intuitiveness. NKDE adopts the proximity along road network instead of the Euclidean distance for estimating the density fields, which can correctly compute the impact of events (e.g., traffic accidents and taxi pick-ups/drop-offs) distributed along the road network. However, this kind of density estimation can only measure the density propagation along a road network that provides 1D measurements [48], unlike the density fields over a 2D space. However, most human activities take place at locations not covered by road networks in a city. NKDE fails to provide an intuitive visualization for density fields at these locations. Taking $p_{1}$ in Fig. 2 b) as an example, users cannot retrieve its density field with NKDE. The lack of density fields hinders certain analytical tasks, such as the comparison of the density fields at $p_{1}$ and at $p_{2}$. Therefore, NKDE can only present the density fields along the road network, whilst users typically need to visualize density fields over an entire urban area. The visualization is unintuitive, making urban analysis less effective.

In summary, there is an urgent need for a new density estimation method that can simultaneously fulfill the requirements of correctness and intuitiveness in urban data visualization and analysis.

\subsection{Overview of Topology Density Map}

We develop the topology density map, a new density estimation method specifically designed for urban data visualization and analysis. Topology density map fulfills the correctness requirement by incorporating road topology and traffic condition into account, and the intuitiveness requirement by extending the density estimation from a $1 \mathrm{D}$ road network to a $2 \mathrm{D}$ planar surface.

Fig. 3illustrates the construction process of the topology density map. The method takes a road network $G$ with vertices $A, B, C$, and $D$, and two POIs $H_{1}$ and $H_{2}$ as inputs (Fig. 3 a)). Thanks to the open data campaign, these urban data are becoming increasingly ubiquitous, such as the open street map (OSM) for road networks. With the information, we can construct a directed acyclic graph (DAG) for each 


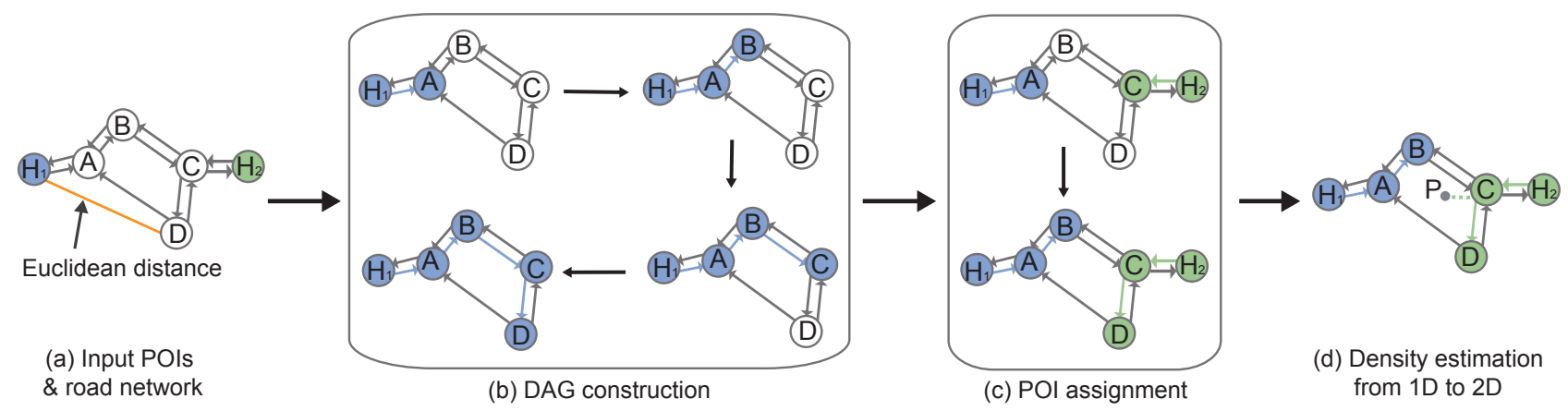

Fig. 3. Overview of topology density map construction. (a) Given a road network (with vertices $A, B, C$, and $D$ ) and multiple POls $\left(H_{1}\right.$, and $\left.H_{2}\right)$, we first (b) compute a directed acyclic graph (DAG) for each POI taking road topology and traffic conditions into account. (c) Next, we assign each vertex to a POI with the highest density field. Here $A \& B$ are assigned to $H_{1}$, while $C \& D$ are assigned to $H_{2}$. (d) Finally, we extend density estimation from 1D road network to $2 \mathrm{D}$ planar surface.

POI, with the POI as the starting vertex and all intersections of the road network connected (Fig. 3. b)). Here, the edges in the road network are bidirectionally modeled, and the connectivity between neighboring vertices is affected by traffic conditions. Thus, the proximity computation is consistent with the reality. For instance, the proximity from POI $H_{1}$ to vertex $D$ is computed as an aggregation of $d_{G}(H A), d_{G}(A B)$, $d_{G}(B C)$, and $d_{G}(C D)$ rather than Euclidean distance between $H$ and $D$ (the yellow line in Fig. 3(a)). Next, we assign each intersection to a POI with the highest density field (Fig. 3 ( $\mathrm{c}$ )). Here $A$ and $B$ are assigned to $H_{1}$, while $C$ and $D$ are assigned to $H_{2}$. Finally, we extend the density estimation from a $1 \mathrm{D}$ road network to a $2 \mathrm{D}$ planar surface (Fig. 3(d)). For point $P$ in the region, we retrieve the shortest path to each $\mathrm{POI}$ by iterating on the neighboring intersections. For instance, the proximity from $H_{1}$ to $P$ is $d_{G}(H A)+d_{G}(A P)$, while that from $H_{2}$ to $P$ is $d_{G}\left(H_{2} C\right)+d_{G}(C P)$. We choose the smaller proximity from $\mathrm{H}_{2}$ to $P$, and compute the density field using that value.

\section{Domain Problem and Data Preparation}

This section first introduces the domain problem of accessibility measurement for public service facilities (Sec. 4.1). Next, we introduce the data used in the study, and describe the data preparation for topology density map construction (Sec. 4.2).

\subsection{Accessibility}

Providing easy access to public service facilities has always been a high-priority topic in urban planning. Here, each public service facility is regarded as a POI. POI accessibility is represented as scalar fields distributed over space, which can be visualized using a density map. Short et al. [36] pointed out that accessibility in an urban area is better computed as access time rather than physical distance. Furthermore, commuting by vehicles is the most common travel mode in cities. Hence, many studies (e.g., [20 34] ) used vehicle access time to indicate POI accessibility.

This work also employs vehicle access time on roads as an index to indicate POI accessibility. A long access time between a source POI and a target location indicates low POI accessibility, and vice versa. As illustrated in Fig. 3, the path from POI $H$ to location $P$ can be modeled as $n$ road segments $\left\{\operatorname{seg}_{i}\right\}_{i=1}^{n}$ and walkway $w$ between $P$ and a surrounding intersection. Hence we can compute POI accessibility Acc of $H$ at location $P$ as:

$$
\operatorname{Acc}(P, H)=\left(\sum_{i=1}^{n} t\left(\operatorname{seg}_{i}\right)+t(w)\right)^{-\alpha}
$$

where $t(\cdot)$ denotes the vehicle access time on a road segment $\operatorname{seg}_{i}$ or the travel time spent on walkway $w$, and $\alpha$ is the accessibility attenuation coefficient that measures the accessibility decay speed. Peeters and Thomas [30] summarized that $\alpha$ typically lies in the range [0.9, 2.29], where small values are preferred for large accessibility ranges, while large values are chosen for small accessibility ranges. This work adopts

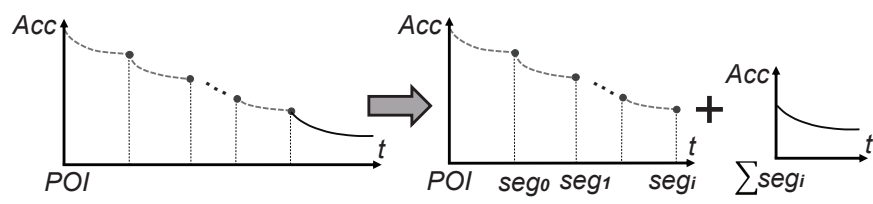

(a)

(b)

(c)

Fig. 4. The overall accessibility (a) between a POI and a location can be divided into (b) accessibility measured on road segments and (c) accessibility measured on walkway from intersection to the location.

a small attenuation coefficient of 1 for large accessibility ranges. Notice also that $t\left(\operatorname{seg}_{i}\right)$ is a time-variant function that is dependent on the dynamic traffic conditions of road segment $\operatorname{seg}_{i}$.

Fig. 4 illustrates the relationship between accessibility ( $y$-axis) and access time ( $x$-axis). The overall accessibility (Fig. 4/a)) can be decomposed into two parts: the accessibility measured on road segments (Fig. 4 b)), and the accessibility measured on a walkway from a surrounding intersection to an arbitrary location in the region (Fig. 4. Given the nature of the traffic data used in the work, we can only compute the average vehicle speed for each road segment. In consideration of this fact, we only compute the accessibility value for each intersection, but not for any point on a road segment. In contrast, we can compute the accessibility value for any point along the walkway. Hence, we use dash lines for road segment accessibility and solid line for walkway accessibility. Notice that both road segment and walkway accessibilities are inversely proportional functions of access time. The function slopes are determined by accessibility attenuation coefficient $\alpha$, which we set to 1 for a gradual decay effect.

\subsection{Data Preparation}

We illustrate the applicability of topology density map by visualizing and analyzing the accessibility for hospitals in Shenzhen, China.

Data Collection. Our topology density map is constructed from three datasets: i) We extract geographical data and the road network from the open street map (OSM) ${ }^{1}$, which is also utilized for our map visualization. ii) We acquire traffic data from an open data platform 2 of Shenzhen, China. We deduce dynamic traffic conditions from the traffic data. iii) We retrieve hospital locations from the Baidu Map Service 3 The hospitals are POIs analyzed in case studies.

Road Segmentation. We define road segment as a part of the road between two adjacent intersections. We treat each road segment, instead of the road (represented as "way" in OSM), as the unit of accessibility analysis in this study. This introduces several advantages: First, by dividing a long road into several road segments, we can deduce more accurate access times because different road segments typically share different speeds. Second, we can easily establish a DAG, in which an

\footnotetext{
https://www.openstreetmap.org/

2 https://opendata.sz.gov.cn/data/dataSet/toDataDetails/29200_00403590

3 https://api.map.baidu.com/lbsapi/getpoint/index.html
} 
Density estimation on road network

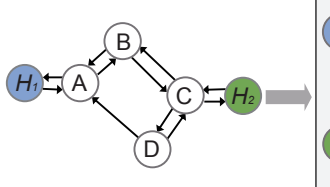

(a) Input Data

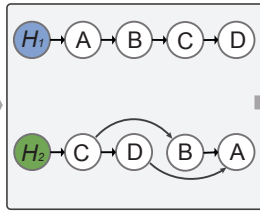

(b) Construct DAGs

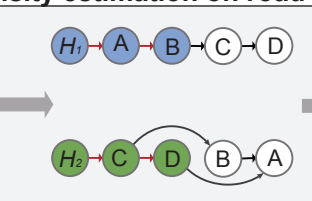

(c) Find the

shortest paths

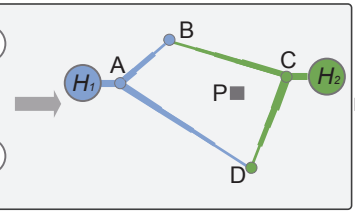

(d) Compute density field on each vertex

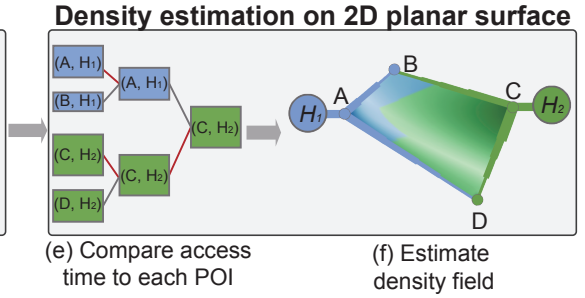

Fig. 5. Topology density map construction: (a) An example of inputs with $H_{1}$ and $H_{2}$ as POls, and a road network with vertices $A, B, C, D$. (b) For each POI, we first construct a DAG that starts from the POI and connects all network vertices. (c) We can easily find the shortest path from the POI to all vertices with the DAG. (d) We can then compute the density field for each vertex. (d) Next, we extend the density estimation from road network to 2D planar surface. (e) Finally, we compute density fields for all points in the region and construct a topology density map.

edge represents a road segment and a vertex represents an intersection. To accomplish road segmentation, we find all intersections in a road network, and group a sequence of edges connecting those vertices as one unit corresponding to a road segment. By combining road segments with traffic data, we can generate an average speed for each road segment in a certain time period.

POI-Intersection Connection. To study how POI accessibility propagates along a road network, we need to connect POIs to road network. POIs have three possible locations relative to a road network in an urban area: right on an intersection, just beside a road, or neither beside any road nor on any intersection. In the first situation, we can simply treat a POI as a vertex in the road network. For the other situations, Weng et al. [45] created a new vertex that is the projection point of a POI to its nearest road in the road network. In contrast, we connect a POI to its nearest intersection. We do this for two reasons. First, due to the availability of input data, traffic conditions and road topology in the planar surface enclosed by a road network are unknown to us. A marginal difference exists between projecting a POI to its nearest road and connecting it directly to the nearest intersection. Second, we can only measure the average speed of each road segment, whilst a vehicle speed is non-uniform when driving on a road. Hence, we cannot measure the exact access time from the projection point to the corresponding intersection.

\section{Topology Density MaP}

In this section, we introduce the key steps on how to construct a topology density map: first, to estimate the density fields on road network (Sec. 5.1, then extend density field estimation to a 2D planar surface (Sec. 5.2). The construction process is illustrated in Fig.5 where the inputs (Fig. 5]a)) include POIs $H_{1} \& H_{2}$, and road network $G$ with vertices $\{A, B, C, D\}$. The goal is to estimate a density field for arbitrary point $P$ within polygon ABCD. Next, we present the visual effects of the topology density map (Sec. 5.3), and its advantage over NKDE in terms of intuitiveness (Sec. 5.4).

\subsection{Density Estimation on Road Network}

In this step, we estimate the density propagation along the road network from POIs, as follows:

Construct DAGs (Fig. 5(b)). After retrieving the access times for the road segments and connecting the POIs to the road network, we construct a DAG for each POI to facilitate the accessibility propagation estimation. Each DAG starts from the corresponding POI, and connects all intersections by directional road segments. Here, vertices correspond to intersections, and edges correspond to road segments. We also consider the road direction, so the edge direction indicates the travel direction of the corresponding road segment. Fig. 5.b) presents two example DAGs for POIs $H_{1}$ and $H_{2}$ in blue and green, respectively. Notice that $H_{1}$ is first connected to vertex $A$ as the nearest intersection, while $\mathrm{H}_{2}$ is first connected to vertex $C$ as the nearest intersection. All vertices in the road network are eventually included in the DAGs, with differences in vertex orders that reflect the road topology.

Find the shortest paths (Fig. 5.c)): Given a DAG, we can easily find the shortest paths from a POI to all vertices using the Dijkstra algorithm. All edges here are weighted according to the access time retrieved from the traffic data. For each vertex, we select the POI with the least access time as the most accessible POI. This has practical significance, as people typically choose the most accessible POI and ignore others in reality. Fig. 5(b) indicates that the most accessible POI for $A$ and $B$ is $H_{1}$, while that for $C$ and $D$ is $H_{2}$.

Compute density field on each vertex (Fig.5(d)): After identifying the most accessible POI for each intersection, we can then compute the density propagation along the road network. Here, we use the color of the most accessible POI to encode an intersection, that is, $A \& B$ are colored blue, while $C \& D$ are colored green. Moreover, to better depict density propagation process on the road network, we further encode road segments with tapered lines that are preferred for graph readability [17]. Here, a line is colored according to its connecting vertex with a high accessibility value, and the line width gradually decreases from the vertex with a higher accessibility value to the other vertex with a lower accessibility value. Taking road segment $B C$ for example, the line color is green for vertex $C$, and the line width is decreases from $C$ to $B$. Users can easily deduce that vertex $C$ has a higher accessibility to $H_{2}$ than vertex $B$ 's accessibility to $H_{1}$.

\subsection{Density Estimation on 2D Planar Surface}

In this step, we extend the density estimation from a 1D road network to a 2D planar surface to fulfill the intuitiveness requirement. Here, we illustrate the process with density estimation for an arbitrary point $P$ as in Fig. 5(d). Due to the lack of road network and traffic condition in area $A B C D$, we adopt a simplified access time measurement in a linear correlation with the Euclidean distance between $P$ and the intersections. Here, we adopt a twofold process as follows:

Compare access time to each POI (Fig. 5. (e)): As discussed in Sec. 4.2 we associate a POI to the road network by connecting the POI to the nearest intersection. Similarly, we also associate an arbitrary point $P$ to road network by connecting $P$ to the neighboring intersections, i.e., $\{A, B, C, D\}$, yielding straight walkways $A P, B P, C P, \& D P$. Given that we have found the most accessible POI for each intersection, we can compute the access time from $P$ to each POI by summarizing the network proximity of each road segment and the Euclidean distance of the straight connection. Taking the access time between $P$ and $H_{1}$ for an example, we have two options: 1) to connect $P$ to $A$, yielding an access time of $\left.t\left(H_{1} A\right)+t(A P) ; 2\right)$ to connect $P$ to $B$, yielding an access time of $t\left(H_{1} A\right)+t(A B)+t(B P)$. Given that the access time on the road network is typically shorter than that on walkways (travel by vehicle $v s$. by walking), we select option 1 . Similarly, we can also compute the access time between $P$ and $H_{2}$ as $t\left(H_{2} C\right)+t(C P)$. The comparison of the access times to $H_{1}$ and $H_{2}$ indicates that the access time to $H_{2}$ is lower, and hence the accessibility to $\mathrm{H}_{2}$ is higher.

Estimate density field (Fig. 5 f)): Hence, we can estimate a density field for $P$ with respect to $\mathrm{H}_{2}$ as:

$$
\lambda\left(P \mid H_{2}, G\right)=\frac{1}{r} K\left(\frac{\left(t\left(H_{2} C\right)+t(C P)\right)^{-\alpha}}{r}\right)
$$

where $\lambda\left(P \mid H_{2}, G\right)$ denotes the density field at $P$ given POI $H_{2}$ and road 


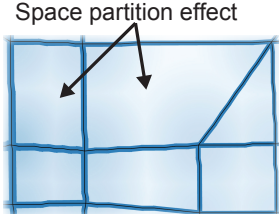

(a) One POI

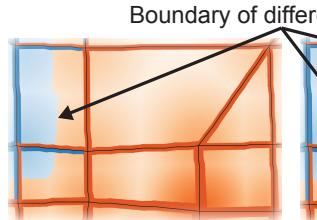

(b) Two POls

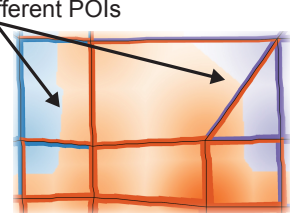

(c) Three POls
Fig. 6. Visual effects of topology density map: color values clearly reveal quantitative density fields distributed in the space when there is only one $\mathrm{POI}$ (a); and additional color hues depict accessibility to different POIs when there are two (b) and three (c) POIs.

network $\left.G, t\left(H_{2} \mathrm{C}\right)+t(C P)\right)^{-\alpha}$ reflects the $\mathrm{H}_{2}$ accessibility at location $P$ (Equation 3), and $K$ is the kernel function with $r$ as the kernel radius (Equation 2). The color of the point is assigned in accordance with the color of the source POI, i.e., $\mathrm{H}_{2}$. Similarly, we can estimate the density fields for other points in the 2D planar surface. Then, we render each pixel corresponding to its density field and the source POI to generate a topology density map. An example of the generated topology density map is presented in Fig. 5(f).

\subsection{Visual Effects of Topology Density Map}

Topology density map adopts color hues to encode different POIs, and color values to indicate quantitative density fields. In this way, users can not only distinguish which POI is the easiest accessible, but also how much easy to access the POI from arbitrary locations in a 2D planar space. In each region segmented by road segments, the method generates a visual effect corresponding to space partition by a weighted Voronoi diagram where the centers are intersections and the weights are network-based proximity to POIs; see Fig. 6.a) for an example. Users can easily identify which intersection is the easiest accessible from a location in the region.

Fig. 6(a-c) shows visual effects of topology density map with of one to three POIs, respectively. There is only one POI in Fig. 6(a), so all density fields are colored in blue. Meanwhile, one can notice that colors are gradually fading from intersections to region centers, indicating those locations near by intersections are easier accessible. When more than one POIs are presented, there will be multiple color hues in the topology density map, e.g., two hues for two POIs in Fig.6 b), and three hues for three POIs in Fig. 6.c). One can easily identify boundaries between different color hues, separating the space into regions by the easiest accessible POIs. Here we can notice that the region is mostly affected by the orange POI. Notice that the space partition effect and boundaries between two POI regions may be affected by various parameters when constructing topology density map, e.g., bandwidth and kernel function. The parameter settings will be further discussed in Sec.7.1
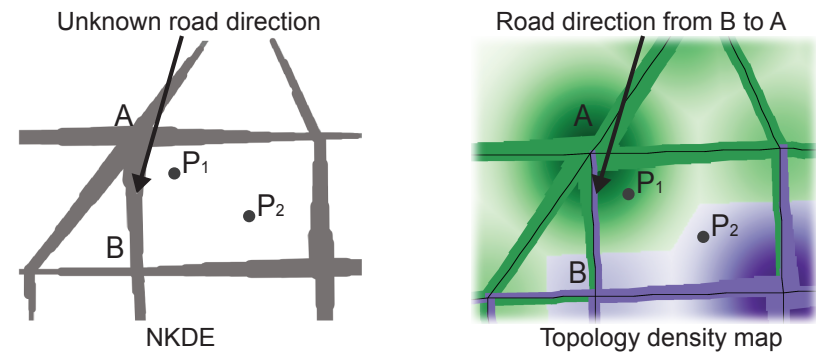

Fig. 7. Comparing topology density map (right) with NKDE (left). Topology density map is more intuitive than NKDE in presenting density distribution over 2D space and depicting road directions.

\subsection{Comparison with NKDE}

Planar KDE and NKDE are state-of-the-art approaches for density estimation. However, planar KDE is inaccurate for urban data analysis and visualization because it omits the road network. In contrast, NKDE estimates density based on the linear propagation along a 1D road network, which has been applied to analyze taxi pick-ups/drop-offs [31. 47] and traffic accidents [13]. Hence we only compare our topology density map with NKDE in terms of intuitiveness (R2).

Fig. 7 presents a comparison of the visualizations generated by NKDE (Fig. 7) left)) and our topology density map (Fig. 7 (right)). Both visualizations are built from the same POIs and local structure of a road network. Here, our topology density map reveals the density fields over the entire space, while NKDE only depicts the density fields along the roads. For instance, the density values for $P_{1} \& P_{2}$ are unknown in Fig. 7 (left). In contrast, the most accessible POI for $P_{1}$ is the green POI, while that for $P_{2}$ is the purple POI; and density value for $P_{1}$ is higher than that for $P_{2}$, from Fig. 7 (right). In this sense, our topology density map supports better the functions of lookup and comparison than NKDE. Moreover, our topology density map also depicts directions of road segments using tapered lines, whilst NKDE only presents density fields along road segments without direction information. Taking the road segments between intersections $A$ and $B$ in Fig. 7 (right) for an example, $A$ is more accessible to the green POI since $A$ is colored green, while $B$ is more accessible to the purple POI. One can also figure out that the road segment on the right side is from $B$ to $A$, while the one on the left side is from $A$ to $B$, by checking either colors of the road segments or taper directions.

In summary, our topology density map surpasses the performance of NKDE in presenting the density fields over 2D space and depicting road directions.

\section{Case Studies ANd Expert InTERView}

We conduct two case studies to verify whether the topology density map satisfies the requirements discussed in Sec. 3.2. We select the Futian district in Shenzhen, China as the study area. The area is presented as the main view in Fig. 1 Feedbacks from three independent domain experts are presented at the end.

\subsection{Study 1: Analyzing the Influence of Road Topology and Traffic Conditions on Accessibility}

We first verify whether the topology density map can present the density fields accurately (requirement R1 as in Sec. 3.2, by taking both road topology and traffic conditions into consideration.

Road Topology. We first zoom in to the selected region shown in Fig. 1.a). The two different colors indicate that this region is easily accessible to two POIs denoted as $H_{1}$ (red color) and $H_{2}$ (blue color). In Fig. 1 a), Regions $1(B C D E)$ and $2(A B E F)$ are easily accessible to both $H_{1}$ and $H_{2}$, while Region $3(D F G I)$ is fully covered by POI $H_{2}$. From the density distribution along the road segments, we can find reasons for the phenomena: intersections $A, B$, and $C$ are easily accessible to POI $H_{1}$, while intersections $D, E$, and $F$ are easily accessible to POI $H_{2}$. Moreover, $H_{2}$ propagates along paths $F-E-B$ and $E-D-C$. To explore the influence of road topology, we generate a new density map by removing paths $F-E$ and $E-D$. Fig. 1 (b) shows the result, which is the same region as that in Fig. 1 (a). The comparison of Figs. 1 b) with 1 a) indicates the changes in density field distribution. Region 1, which is easily accessible to both $H_{1}$ and $H_{2}$ in Fig. 11 (a), is now fully covered by POI $H_{1}$ in Fig. 11(b). In contrast, Region 3, which is previously only covered by POI $\mathrm{H}_{2}$ in Fig. 1 a), is now accessible to both $H_{1}$ and $H_{2}$ in Fig. 1 b).

Removing paths $F-E$ and $E-D$ makes path $F-E-D$ inaccessible, yielding changes to road topology. Therefore, the previous path $H_{2}-F-E-D$ with the shortest access time is no longer available, changing the most accessible POI at intersection $D$ from $H_{2}$ to $H_{1}$, and that at intersection $E$ from $H_{2}$ to $H_{1}$. This affects the construction of the topology density map in terms of estimating the density propagation from POIs and determining the source POI (Sec. 5.2. Hence, the resulting topology density map can also reflect the changes. In contrast, Euclidean distances between arbitrary locations in the regions and POIs are unaffected by road topology change, resulting in unchanged planar KDE.

\section{Traffic Condition.}




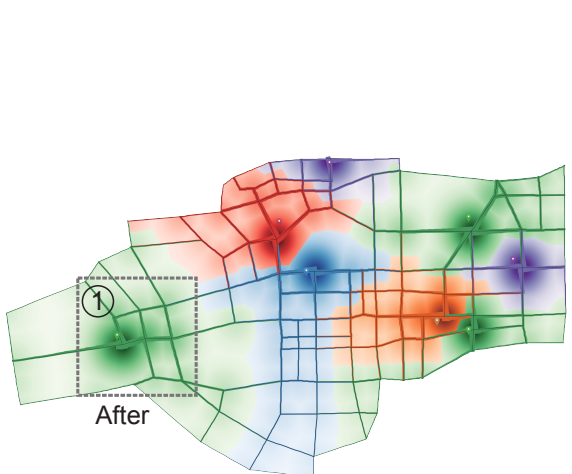

(a) Build a hospital where the density is low

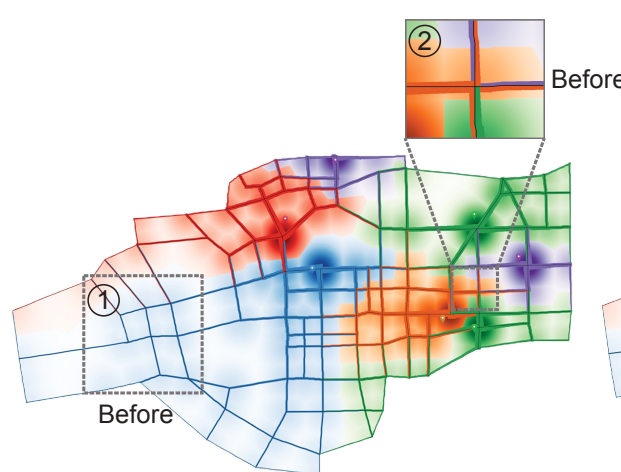

(b) Topology density map for exisiting hospitals

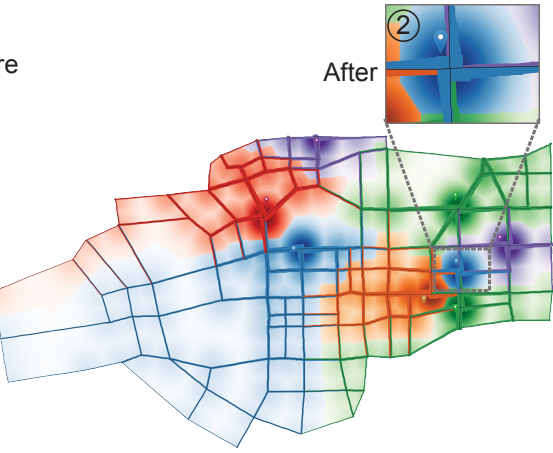

(c) Build a hospital where the density is high

Fig. 8. Topology density map built from existing hospitals (b), after adding a new hospital in region 1 with low density (a), and after adding a hospital in region 2 with high density (c). Region 1 is a preferred location, as density field is more evenly distributed in (a) than in (c).

We then zoom in to the selected region shown in Fig. 11 c). The figure illustrates that the region is easily accessible to two POIs denoted as $H_{3}$ (orange color) and $H_{4}$ (green color). Region 4 is accessible to both $\mathrm{H}_{3}$ and $\mathrm{H}_{4}$, while Region 5 is fully covered by POI $H_{4}$. In general, the density field distribution shows a trend of continuous accessibility propagation toward the outbound of the district. However, when focusing on the green region $\left(H_{4}\right)$, we notice some unusual patterns. A very narrow green part can be observed in Region 4, while the green part increases again in Region 5. The variation of the density fields along the road segments reveals that path $M-N-O$ connects Regions 4 and 5 . We obtain the average speeds of these road segments $(M-N$ and $N-O)$, and find that these segments exhibit high average speeds, reducing the access time through path $M-N-O$ even though the Euclidean distance is long.

To explore the influence of traffic conditions on density fields, we reduce the average speeds of road segments $M-N$ and $N-O$ manually. The density field changes correspondingly, as illustrated in Fig. 1]d) The easiest accessible POI in Region 4 shows no change. However, it changes from $\mathrm{H}_{4}$ to $\mathrm{H}_{3}$ in Region 5 and the density fields decrease as well, caused by lower speeds of road segments $M-N$ and $N-O$. This phenomenon indicates that although the Euclidean distances between intersections remain unchanged, dynamic traffic conditions can lead to variations in shortest paths on a road network and ultimately alter the density distribution on a 2D planar surface.

\subsection{Study 2: Finding the Optimal Location of a New Public Service Facility}

Next, we demonstrate the intuitiveness of topology density map in presenting density distribution (requirement R2 as in Sec. 3.2), which facilitates the process of finding the optimal location of a new public service facility. Here, we use hospitals in Futian District crawled from Baidu Map Service as targeting public service facilities, and access time to measure accessibility to those hospitals. Fig. 8 (b) presents density field of accessibility measured from the hospitals, which is unbalanced in the district as currently the hospitals are concentrated in the east part of Futian District. The government decides to build a new hospital and tries to find a suitable location for the hospital.

A critical consideration here is that the hospital should make the distribution of medical service more balanced. In other words, to maximize the increase of hospital accessibility for the whole district. Two possible options for the hospital location are available.

- First, we can put the new hospital in Region 1 where density is low. The topology density map is updated correspondingly and presented in Fig. 8(a). Obviously, the density field in the southwest part increases, especially that in Region 1. This intuitively depicts how much the region can benefit from a new public service facility. Moreover, because the density first propagates along the road network, and then propagates to a 2D planar surface, the change in density distribution on the road segments is obvious. In short, putting the new hospital in Region 1 balances the accessibility to medical service.

- Second, we can place the hospital in Region 2 where the density is already high. The updated topology density map is shown in Fig. 8.c). Overall, both densities in the selected region and along the surrounding road segments increase. However, this arrangement does not improve the southwest part with low density. Hence, building a new hospital in Region 2 worsens the unbalanced distribution of medical service.

The study clearly demonstrates that the topology density map generates intuitive visualizations that can facilitate problem identification and decision making.

\subsection{Expert Interview}

To evaluate our topology density map, we interviewed three independent domain experts. The first expert (Expert A) is an assistant professor in University S specializing in smart city related research. The second expert (Expert B) is a Ph.D. candidate in University $\mathrm{H}$ whose research focuses on data-driven urban planning. The third expert (Expert C) is a practitioner working in an urban planning institute. Before the interview, we first made a brief introduction of our method, and then explained the details of the two case studies. We collected feedbacks in terms of the feasibility, usability, and effectiveness of our topology density map from the experts in the end. All experts provided valuable feedbacks based on their own background. The detailed feedbacks are summarized below.

Feasibility \& Usability. All three experts agreed that using a density map to reveal accessibility of POIs in an urban area is a good choice. Our topology density map can really help in their research and work. Expert A mentioned that "topology density map takes road topology and traffic conditions into consideration, which fits the reality very well. If the method is applied in my research, it will help me a lot on decision making." Expert B said that "topology density map is of great research value because it considers road structure, and covers the whole city." They all agreed that our method visualizes density field intuitively. Expert B commented that "from the cases you introduced, I can see that topology density map does provide an intuitive visualization, especially in case study 2 . Through topology density map, I can quickly find that the current distribution of hospitals, that is, accessibility to medical resources, is indeed unbalanced. After you manually add a new hospital at two different places, the new density maps show the difference intuitively and definitely help me make a decision."

Effectiveness. All experts thought that the topology density map is an effective tool for urban analysis. Expert $\mathrm{C}$ shared her own experience in identifying suitable locations for new public service facilities. In daily work, her team often need to place a certain number of public service facilities evenly around a few residential blocks, but they do not have an intuitive and easy-to-use tool that could assist them in finding optimal locations. Currently, the team rely on manual approaches: they 


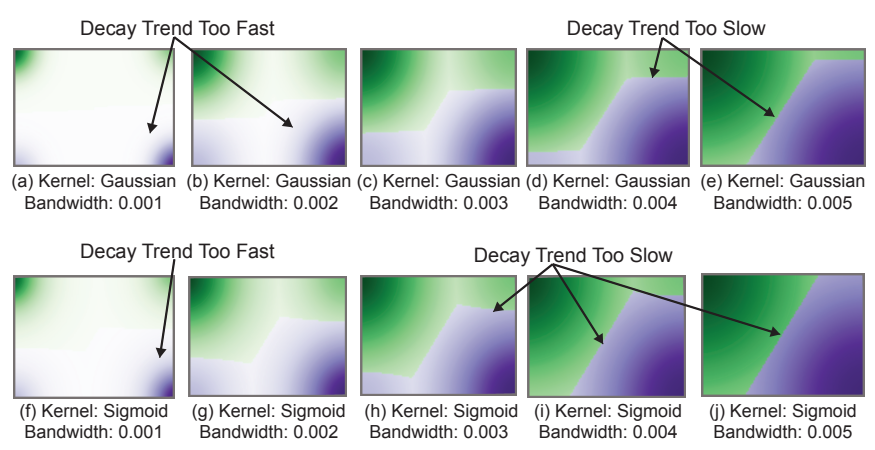

Fig. 9. Parameter selection: (a) to (e) are Gaussian kernel function with different bandwidths, and (f) to (j) are Sigmoid kernel function with different bandwidths.

first mark streets that are close to the residential area, then measure distance from the residential area to each street, and finally draw contour lines based on the measured distances. The process is rather ineffective, and often leads to unsatisfactory results since the distance measurement omits traffic conditions. Moreover, they also find it is difficult to share the planning with others, and would be impossible for others to make modifications. She believes that the topology density map is the right tool they are looking for, not only for finding locations of public service facilities, but also for comparing various planning strategies.

Suggestions. The experts also gave fruitful suggestions on how to improve the topology density map. Both Experts A and C commented that developing our method into a mature software that supports interactive planning would be great. As potential end users, urban planners typically have little knowledge of programming. Expert B pointed out that the unavailability of non-uniform travel speeds in a road segment affects the accuracy of our method in density estimation. She suggested that if we get more detailed data, we can consider connecting POIs to road segments directly, rather than to the nearest intersection because the vehicle speeds on a single road segment can vary dramatically. Expert A also suggested that after solving the problem of insufficient and inaccurate data, we can consider some demographic factors, such as population distribution, which are important elements to be considered in urban analysis. For instance, in case study 2, a more practical strategy is to consider population distribution over space as well.

\section{Discussion}

We next discuss parameter selection (Sec.7.1), computation efficiency (Sec.7.2), and alternative designs (Sec.7.3) for the topology density map. We then discuss the limitations, and summarize the future work in the end (Sec. 7.4.

\subsection{Parameter Selection}

As discussed in Sec.5.2 a kernel function is used as a decay factor when we estimate density on a 2D planar surface. Therefore, a reasonable decay function is particularly important for topology density map. A reasonable decay effect can not only yield an intuitive visualization, but also help users understand the density distribution in urban areas and facilitate decision-making. In contrast, a slow or fast decay parameter will increase the difficulty of observing the density distribution.

Two key parameters in KDE, i.e., kernel function $K$, and bandwidth $r$, affect the decay speed. Many studies [3|28 29 32] suggest that choosing a proper bandwidth is more important than a kernel function. Therefore, we only compare two widely used kernel functions - Gaussian and Sigmoid. For each kernel function, we further compare five equally spaced bandwidths from 0.001 to 0.005 . Bandwidth $r$ corresponds to the accessibility value as in Equation 3 measured between the target point on the 2D planar surface and the source POI. We compute the density estimation when the accessibility value is within the range; otherwise, we set the density value to 0 . We aim to find a good combination of kernel function and bandwidth for this study.

The comparison result is shown in Fig. 9 Each sub-figure presents the density field of the region enclosed by the rectangle. The vertices of the rectangle represent intersections, and edges represent road segments in a road network. In each rectangle, two different POIs are positioned at the top-left (green POI) and bottom-right (purple POI) vertices. Here, we only compare differences of density distribution within the planar surface computed from different parameters, so the density distribution on road segments is not taken into consideration. Fig.9 indicates that for the same POIs and road topology, different combinations of kernel function and bandwidth produce different visual effects. In Fig.99 a,b,f), the density decay is too fast, making it difficult to observe the density change, especially for points in the middle of each rectangle. Consequently, it is difficult for users to perceive accessibility differences. In Fig. 9. d,e,h,i,j), the density decay is too slow to distinguish the density values. The results suggest to discard these parameter combinations. In addition, both Gaussian and Sigmoid kernels show similar trends of decaying too slow with small bandwidths, whilst too fast with big bandwidths. The results reveal that different kernel functions have no significant impact on density estimation, but different bandwidths do. This finding is consistent with those of previous studies [3, 28, 29, 32].

On the basis of the results in Fig. 9 we eventually choose Gaussian as the kernel function and 0.003 as the bandwidth. All experiments and case studies in this work are conducted using these parameters. Nevertheless, the visual effects are also determined by many factors, including accessibility measurement, color perception, and study areas. Different application scenarios may have different requirements for decay effect. The purpose of this paper is to illustrate an accurate and intuitive method for density map generation. Users can make their own choices in different cases.

Table 1. Running time (in milliseconds) comparison for topology density map and planar KDE by different number of POls and image size.

\begin{tabular}{|c|l|l|l|l|l|l|}
\hline \multirow{2}{*}{ Image Size } & \multicolumn{2}{|c|}{10 POIs } & \multicolumn{2}{c|}{ 50 POIs } & \multicolumn{2}{c|}{100 POIs } \\
\cline { 2 - 7 } & Topology & Planar & Topology & Planar & Topology & Planar \\
\hline $1920 \times 1200$ & 2010.75 & 99.41 & 1992.04 & 144.21 & 2134.92 & 192.20 \\
\hline $1280 \times 800$ & 962.55 & 51.25 & 971.14 & 78.49 & 991.41 & 116.29 \\
\hline $640 \times 400$ & 353.87 & 18.98 & 381.97 & 24.33 & 347.03 & 32.70 \\
\hline
\end{tabular}

\subsection{Computation Efficiency}

NKDE only estimates the density along a 1D road network, and often the density fields are estimated using average travel speeds of road segments. In contrast, topology density map and planar KDE provide density estimation over an entire 2D planar surface. Hence, NKDE would require much less computation time than topology density map and planar KDE. For fairness, we only compare the computation efficiency of our approach with planar KDE and not with NKDE. The experiment runs on a MacBook Pro $2.5 \mathrm{GHz}$ Core i7 with a Radeon R9 M370X graphics card, and the density maps are rendered in a front-end web interface using Javascript. To avoid interference of other factors, we adopt the same study area, traffic data, and POIs in both density maps. Our topology density map takes pre-computed DAGs (Sec. 5.1) starting from each POI to all intersections using Dijkstra shortest path algorithm as additional input.

Table 1 shows the running times (in milliseconds) of the topology density map and planar KDE under different numbers of POIs and image sizes. Overall, the table shows that the computation efficiency for planar KDE is affected by both image size and number of POIs, while that for the topology density map is mainly affected by image size. This is consistent with our expectation, as planar KDE needs to measure density fields for 2D space (i.e., the image size) based on input POIs (see Equation 11. Instead, computation cost for the topology density map is mainly spent on extending density fields from road network to 2D space (see Equation 4, and the process is dependent on the number of intersections rather than number of POIs. Moreover, with the same image size and number of POIs, planar KDE requires much less running time than the topology density map, especially when the number of POIs is small. This is because there are in total 273 intersections in the study area, which is much more than that of POIs, and for each point in a region, we need to compute density fields extended from multiple neighboring intersections. 
Note that the running time for the topology density map is measured scratch, which is much slower than that for planar KDE. Nevertheless, in many real-world scenarios (e.g., case study 2 ), we can pre-compute a density estimation for an entire area based on existing POIs, then we only need to re-compute the density fields for a small region affected by a new POI. This process can be finished in real-time since the number of intersections to be updated is small.

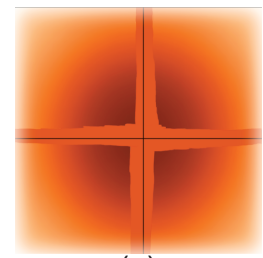

(a)

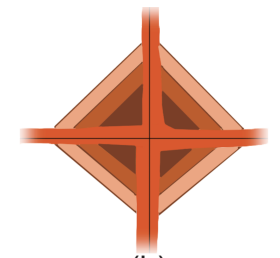

(b)

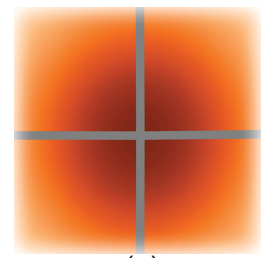

(c)
Fig. 10. Alternative designs to visualize density distribution: (a) topology density map used in this study, (b) a contour-based design, and (c) density map without showing density on roads.

\subsection{Alternative Design}

Two alternative designs for visualizing scalar fields distributed in a 2D space are considered. Fig. 10 b) is our first attempt, which employs tapered lines with line thickness to visualize the density propagation along roads, and a contour map to visualize the density distribution in a 2D planar surface. Each contour represents the region that can be accessed within a certain time. However, after discussing the design choice with a domain expert (Expert C), she pointed out that a contour map can only depict discrete access times. As in Fig. 10.b), there are only three time intervals, and one can only retrieve which places can be accessed within these three different time intervals. We can increase the number of time intervals, but the contour map will become chaotic when contours are dense. Consequently, finding useful information becomes difficult. Moreover, visualizing the density fields is difficult for contour map when multiple POIs exist. Fig. 10 (c) is another design that adopts density map to visualize density fields in a 2D planar surface. The density map is constructed in the same way as our topology density map. However, the design adopts only straight lines to reflect road connections, rather than tapered lines to visualize density propagation along road segments. Without tapered lines, density propagation along roads is difficult for users to follow.

Therefore, we choose topology density map coupled with tapered lines (Fig. 10.a)) as our final design.

\subsection{Limitations and Future Work}

Limitations. The case studies (Secs. 6.1 \& 6.2) and expert feedbacks (Sec.6.3) confirm that topology density map satisfies the correctness and intuitiveness requirements (Sec. 7.4). Nevertheless, improvement can still be made. First, our current implementation does not include the efficiency of generating the density map as the first priority. We need to compute the density value for each pixel in the screen, which is rather time-consuming. Several promising directions for improving the computing efficiency can be taken, such as GPU-based implementation of density computation [24] and space partition using weighted Voronoi diagram [15]. Second, in the case studies, we assume that vehicles drive on a road segment at a constant speed, which is impossible in reality. We make this simplified assumption because a more detailed traffic data is unavailable. As suggested by domain experts, we would like to produce a more accurate density estimation with fine-grained speed information on roads. Third, due to the lack of building and walkway data in each zone, we only consider the direct connection between a location and its surrounding intersections when extending the density estimation from the road network to a planar surface, which again leads to inaccurate estimation. For example, if a zone (e.g., a campus or housing estate) possesses only one entrance, the density estimation for a location in the zone shall include the location's distance to the entrance and the entrance's distance to road network. We would like to fuse other kinds of urban data, such as land use and building types, to generate more accurate density estimation.
Future Work. In addition to improving the efficiency and accuracy of the topology density map construction, many fruitful directions for future work can be taken. First, we would like to integrate topology density map into many real-world applications, e.g., a real-time risk monitoring system. The system can provide interactive visualization for POI accessibility analysis under real-time traffic conditions. We anticipate that such a system can assist policy makers in making appropriate decisions in case of emergency, such as to improve fire truck scheduling strategies in case of fire. Second, providing temporal information in the context of a map view is a always challenging task in geographical information system. Many studies adopt isochrone map, which however can only depict discrete temporal information; see the comparison of topology density map with contour map above. For an example, Zeng et al. [49] used isochrone maps to depict accessible regions within 30 and 60 minutes using public transportation system. However, detailed travel times can only be depicted with an additional isotime flow map view. We plan to integrate temporal information on a map seamlessly using topology density map. Last but not least, this work employs access time as the indicator to reflect POI accessibility. In fact, topology density map can be applied in various scenarios as long as the analyses rely on certain topological structures, e.g., network connectivity. We would like to exploit the potential of applying topology density map in those scenarios.

\section{ConClusion}

In this paper, we have introduced Topology Density Map, a new method for density estimation in the context of an urban environment. Topology density map surpasses the performance of conventional planar KDE and NKDE in satisfying two specific requirements for urban data visualization and analysis: First, the density fields estimation should be correct, in order to support urban analysis and assist decision-making; Second, the visualization should be intuitive that depicts density fields over an entire 2D space instead of only 1D road network. The correctness requirement is achieved by including complex road topology and dynamic traffic conditions into consideration, while the intuitiveness requirement is achieved by extending accessibility to arbitrary points in the 2D space.

To facilitate the construction process of topology density map, we first construct a series of DAGs for input POIs, of which a DAG propagates nonlinear accessibility from the source POI to all intersections in a road network. Next, to extend density field estimation from road network to the entire planar surface, we identify key vertices in each DAG, compute and compare density field for every point assorted with each POI, and finally color code every point according to the computed density field and corresponding POI. Topology density maps exhibit a weighted Voronoi diagram-like visual effect, which divides the 2D planar surface into different regions in accordance with POI accessibility. Various parameters and alternative designs have been considered, and we choose an optimal setting that meets the intuitiveness requirement. Two case studies of exploring hospital accessibility based on real-world traffic data in an urban area, together with positive feedbacks from three independent domain experts, approved the topology density map in terms of feasibility, usability, and effectiveness.

\section{ACKNOWLEDGMENTS}

We thank all the domain experts interviewed in this research. We also thank the reviewers for their comments and suggestions. The work is supported in part by the National Key Research and Development Plans (2019YFC0810705 and 2018YFC0807002) of P. R. China, National Natural Science Foundation of China (No. 61802388), HK RGC GRF grant 16208514 and HKUST SSC grant F0707.

\section{REFERENCES}

[1] G. Andrienko, N. Andrienko, W. Chen, R. Maciejewski, and Y. Zhao. Visual analytics of mobility and transportation: State of the art and further research directions. IEEE Transactions on Intelligent Transportation Systems, 18(8):2232-2249, 2017. 
[2] A. Baddeley, G. Nair, S. Rakshit, G. McSwiggan, and T. M. Davies. Analysing point patterns on networks-a review. Spatial Statistics, p. $100435,2020$.

[3] T. C. Bailey and A. C. Gatrell. Interactive spatial data analysis (vol. 413). Essex: Longman Scientific \& Technical, 1995.

[4] Z. I. Botev, J. F. Grotowski, D. P. Kroese, et al. Kernel density estimation via diffusion. The Annals of Statistics, 38(5):2916-2957, 2010.

[5] W. Chen, F. Guo, and F.-Y. Wang. A survey of traffic data visualization. IEEE Transactions on Intelligent Transportation Systems, 16(6):29702984, 2015.

[6] D. Chu, D. A. Sheets, Y. Zhao, Y. Wu, J. Yang, M. Zheng, and G. Chen. Visualizing hidden themes of taxi movement with semantic transformation. In IEEE Pacific Visualization Symposium, pp. 137-144, 2014.

[7] J. Delso, B. Martín, and E. Ortega. A new procedure using network analysis and kernel density estimations to evaluate the effect of urban configurations on pedestrian mobility. the case study of Vitoria-Gasteiz. Journal of Transport Geography, 67:61-72, 2018.

[8] M. Deng, X. Yang, Y. Shi, J. Gong, Y. Liu, and H. Liu. A density-based approach for detecting network-constrained clusters in spatial point events. International Journal of Geographical Information Science, 33(3):466488, 2019.

[9] Z. Deng, D. Weng, J. Chen, R. Liu, Z. Wang, J. Bao, Y. Zheng, and Y. Wu. Airvis: Visual analytics of air pollution propagation. IEEE transactions on visualization and computer graphics, 26(1):800-810, 2019.

[10] L. Dong, R. Li, J. Zhang, and Z. Di. Population-weighted efficiency in transportation networks. Scientific Reports, 6(1):26377, 2016.

[11] N. Ferreira, J. Poco, H. T. Vo, J. Freire, and C. T. Silva. Visual exploration of big spatio-temporal urban data: A study of new york city taxi trips. IEEE Transactions on Visualization and Computer Graphics, 19(12):2149-2158, 2013.

[12] T. H. Grubesic. On the application of fuzzy clustering for crime hot spot detection. Journal of Quantitative Criminology, 22(1):77, 2006.

[13] H. Harirforoush and L. Bellalite. A new integrated GIS-based analysis to detect hotspots: a case study of the city of Sherbrooke. Accident Analysis \& Prevention, 130:62-74, 2019.

[14] J. He, H. Chen, Y. Chen, X. Tang, and Y. Zou. Diverse visualization techniques and methods of moving-object-trajectory data: a review. ISPRS International Journal of Geo-Information, 8(2):63, 2019.

[15] K. E. Hoff III, J. Keyser, M. Lin, D. Manocha, and T. Culver. Fast computation of generalized voronoi diagrams using graphics hardware. In Proceedings of the 26th annual conference on Computer graphics and interactive techniques, pp. 277-286, 1999.

[16] M. Hogräfer, M. Heitzler, and H.-J. Schulz. The State of the Art in Map-Like Visualization. Computer Graphics Forum, 2020.

[17] D. Holten, P. Isenberg, J. J. van Wijk, and J.-D. Fekete. An extended evaluation of the readability of tapered, animated, and textured directededge representations in node-link graphs. In IEEE Pacific Visualization Symposium, pp. 195-202. Hong Kong, 2011.

[18] C. Hurter, O. Ersoy, S. I. Fabrikant, T. R. Klein, and A. C. Telea. Bundled visualization of dynamicgraph and trail data. IEEE Transactions on Visualization and Computer Graphics, 20(8):1141-1157, 2014.

[19] C. Hurter, B. Tissoires, and S. Conversy. FromDaDy: Spreading aircraft trajectories across views to support iterative queries. IEEE Transactions on Visualization and Computer Graphics, 15(6):1017-1024, 2009.

[20] M. Jin, L. Liu, D. Tong, Y. Gong, and Y. Liu. Evaluating the spatial accessibility and distribution balance of multi-level medical service facilities. International Journal of Environmental Research and Public Health, 16(7):1150, 2019.

[21] I. Kloog, A. Haim, and B. A. Portnov. Using kernel density function as an urban analysis tool: Investigating the association between nightlight exposure and the incidence of breast cancer in Haifa, Israel. Computers, Environment and Urban Systems, 33(1):55-63, 2009.

[22] M.-J. Kraak and F. Ormeling. Cartography: Visualization of Geospatial Data. CRC Press, $4^{\text {th }}$ ed., 2020.

[23] R. Krüger, G. Simeonov, F. Beck, and T. Ertl. Visual interactive map matching. IEEE Transactions on Visualization and Computer Graphics, 24(6):1881-1892, 2018.

[24] O. D. Lampe and H. Hauser. Interactive visualization of streaming data with kernel density estimation. In IEEE Pacific Visualization Symposium, pp. 171-178, 2011.

[25] C. Lee, Y. Kim, S. M. Jin, D. Kim, R. Maciejewski, D. Ebert, and S. Ko. A visual analytics system for exploring, monitoring, and forecasting road traffic congestion. IEEE Transactions on Visualization and Vomputer
Graphics, 2019.

[26] R. Li, L. Dong, J. Zhang, X. Wang, W.-X. Wang, Z. Di, and H. E. Stanley. Simple spatial scaling rules behind complex cities. Nature communications, 8(1):1-7, 2017.

[27] D. Liu, D. Weng, Y. Li, J. Bao, Y. Zheng, H. Qu, and Y. Wu. Smartadp: Visual analytics of large-scale taxi trajectories for selecting billboard locations. IEEE Transactions on Visualization and Computer Graphics, 23(1):1-10, 2016

[28] D. O'sullivan and D. Unwin. Geographic Information Analysis. John Wiley \& Sons, 2014.

[29] D. O'Sullivan and D. W. Wong. A surface-based approach to measuring spatial segregation. Geographical Analysis, 39(2):147-168, 2007.

[30] D. Peeters and I. Thomas. Distance predicting functions and applied location-allocation models. Journal of Geographical Systems, 2(2):167184, 2000.

[31] T. Pei, W. Wang, H. Zhang, T. Ma, Y. Du, and C. Zhou. Density-based clustering for data containing two types of points. International Journal of Geographical Information Science, 29(2):175-193, 2015.

[32] O. Schabenberger and C. A. Gotway. Statistical Methods for Spatial Data Analysis. CRC press, 2017.

[33] R. Scheepens, N. Willems, H. van de Wetering, G. Andrienko, N. Andrienko, and J. J. van Wijk. Composite density maps for multivariate trajectories. IEEE Transactions on Visualization and Computer Graphics, 17(12):2518-2527, 2011.

[34] R. Scheepens, N. Willems, H. van de Wetering, and J. J. van Wijk. Interactive density maps for moving objects. IEEE Computer Graphics and Applications, 32(1):56-66, 2012.

[35] Y. Shen, L. Zhao, and J. Fan. Analysis and visualization for hot spot based route recommendation using short-dated taxi GPS traces. Information, 6(2):134-151, 2015.

[36] M. B. Short, A. L. Bertozzi, and P. J. Brantingham. Nonlinear patterns in urban crime: Hotspots, bifurcations, and suppression. SIAM Journal on Applied Dynamical Systems, 9(2):462-483, 2010.

[37] B. W. Silverman. Density estimation for statistics and data analysis. Routledge, 2018.

[38] G. Sun, R. Liang, H. Qu, and Y. Wu. Embedding spatio-temporal information into maps by route-zooming. IEEE transactions on visualization and computer graphics, 23(5):1506-1519, 2016.

[39] M. Talebi, B. Majnounian, M. Makhdoum, E. Abdi, M. Omid, E. Marchi, and A. Laschi. A GIS-MCDM-based road network planning for tourism development and management in Arasbaran forest, Iran. Environmental Monitoring and Assessment, 191(11):647, 2019.

[40] L. Tang, Z. Kan, X. Zhang, F. Sun, X. Yang, and Q. Li. A network kernel density estimation for linear features in space-time analysis of big trace data. International Journal of Geographical Information Science, 30(9):1717-1737, 2016.

[41] D. Thom, H. Bosch, S. Koch, M. Wörner, and T. Ertl. Spatiotemporal anomaly detection through visual analysis of geolocated twitter messages. In IEEE Pacific Visualization Symposium, pp. 41-48, 2012.

[42] W. R. Tobler. A computer movie simulating urban growth. Economic Geography, 46:234-240, 1970.

[43] C. Tominski, H. Schumann, G. Andrienko, and N. Andrienko. Stackingbased visualization of trajectory attribute data. IEEE Transactions on Visualization and Computer Graphics, 18(12):2565-2574, 2012.

[44] D. Weng, C. Zheng, Z. Deng, M. Ma, J. Bao, Y. Zheng, M. Xu, and Y. Wu. Towards better bus networks: A visual analytics approach. arXiv preprint arXiv:2008.10915, 2020.

[45] D. Weng, H. Zhu, J. Bao, Y. Zheng, and Y. Wu. Homefinder revisited: Finding ideal homes with reachability-centric multi-criteria decision making. In Proceedings of the CHI Conference on Human Factors in Computing Systems, pp. 1-12, 2018.

[46] N. Willems, H. van de Wetering, and J. J. van Wijk. Visualization of vessel movements. Computer Graphics Forum, 28(3):959-966, 2009.

[47] Z. Xia, H. Li, Y. Chen, and W. Liao. Identify and delimitate urban hotspot areas using a network-based spatiotemporal field clustering method. ISPRS International Journal of Geo-Information, 8(8):344, 2019.

[48] Z. Xie and J. Yan. Kernel density estimation of traffic accidents in a network space. Computers, Environment and Urban Systems, 32(5):396406, 2008.

[49] W. Zeng, C.-W. Fu, S. M. Arisona, A. Erath, and H. Qu. Visualizing mobility of public transportation system. IEEE Transactions on Visualization and Computer Graphics, 20(12):1833-1842, 2014.

[50] W. Zeng, C.-W. Fu, S. M. Arisona, S. Schubiger, R. Burkhard, and K.-L. 
Ma. Visualizing the relationship between human mobility and points of interest. IEEE Transactions on Intelligent Transportation Systems, 18(8):2271-2284, 2017.

[51] W. Zeng, Q. Shen, Y. Jiang, and A. Telea. Route-aware edge bundling for visualizing origin-destination trails in urban traffic. Computer Graphics Forum, 38(3):581-593, 2019.

[52] P. Zhao, X. Liu, J. Shen, and M. Chen. A network distance and graphpartitioning-based clustering method for improving the accuracy of urban hotspot detection. Geocarto International, 34(3):293-315, 2019. 